\title{
Title: Comparison of 2D vs M-mode echocardiography for assessing fetal myocardial wall
} thickness

Running title: $2 \mathrm{D}$ vs M-mode for fetal cardiac walls thickness

Authors: Alvaro Sepúlveda-Martínez, ${ }^{1,2}$ Laura García-Otero, ${ }^{1}$ Iris Soveral, ${ }^{1}$ Laura Guirado, ${ }^{1}$ Brenda Valenzuela-Alcaraz, ${ }^{1}$ Ximena Torres, ${ }^{1}$ Mérida Rodriguez-Lopez, ${ }^{1}$ Eduard Gratacos, ${ }^{1}$ Olga Gómez, ${ }^{1 *}$ Fàtima Crispi. ${ }^{1}$

${ }^{1}$ Fetal i+D Fetal Medicine Research Center, BCNatal - Barcelona Center for Maternal-Fetal and Neonatal Medicine (Hospital Clínic and Hospital Sant Joan de Deu), Institut Clínic of Gynecology, Obstetrics and Neonatology, IDIBAPS, Universitat de Barcelona, CIBER-ER, Barcelona, Spain.

${ }^{2}$ Fetal Medicine Unit, Department of Obstetrics and Gynecology Hospital Clínico Universidad de Chile. Santiago de Chile.

Manuscript word count: 2503

Table count: 5

Figures count: 4

*Corresponding author:

Olga Gomez, MD PhD.

BCNatal - Barcelona Center for Maternal-Fetal and Neonatal Medicine.

(Hospital Clínic and Hospital Sant Joan de Deu).

Sabino de Arana 1, 08028 Barcelona, Spain.

Telephone: +34 93227 9333; Fax: +34 932275612 .

E-mail: ogomez@clinic.cat

Conflict of interest statement: No conflict of interest. 
Funding statement: This project has been co-funded with support of the Erasmus Mundus Joint Doctorate in Fetal and Perinatal Medicine Programme of the European Union (Framework Agreement number: 2013-0040). This publication reflects the views only of the author, and the Commission cannot be held responsible for any use which may be made of the information contained therein. Additionally, the research leading to these results has received funding from "la Caixa" Foundation; Cerebra Foundation for the Brain Injured Child (Carmarthen, Wales, UK); the Instituto de Salud Carlos III (PI14/00226, PI15/00263, PI15/00130 and INT16/00168) as part of the Plan Nacional de I+D+I and co-funded by ISCIII-Subdirección General de Evaluación y el Fondo Europeo de Desarrollo Regional (FEDER) "Otra manera de hacer Europa" (Spain) and the support of the Secretaria d'Universitat i Recerca del Departament d'Economia i Coneixement de la Generalitat de Catalunya (Grant SGR n ${ }^{\circ} 928$ and 2016FI_B01184). Iris Soveral was supported by a grant (Ajut Josep Font) from Hospital Clinic Barcelona.

\section{Bulleted statements:}

What's already known about this topic? There is no consensus agreement of how to measure fetal myocardial walls. A direct comparison between M-mode and 2D-mode in fetal heart assessment has never been described.

What does this study add? We provide a strict methodology for the measurement of fetal myocardial walls and demonstrate that both $2 \mathrm{D}$ and $\mathrm{M}$-mode can be used in a reproducible manner for measuring fetal myocardial wall thickness, with a slightly better performance of M-mode. 


\begin{abstract}
Objective: M-mode and 2D have been proposed for evaluating fetal myocardial thickness. However, studies comparing the performance of both modalities are lacking. We aimed to compare $2 \mathrm{D}$ vs $\mathrm{M}$-mode reproducibility for assessing myocardial wall thicknesses.

Methods: A prospective study including 45 healthy fetuses from low-risk pregnancies evaluated between 18 and 41 weeks of gestation. Left and right ventricular free-wall and septal myocardial thicknesses were measured at end-diastole (ED) and end-systole (ES) in transverse 4-chamber view using $2 \mathrm{D}$ and $\mathrm{M}$-mode. Intra- and inter-observer reproducibility was evaluated by the concordance correlation coefficient (CCC). Both techniques were compared by t-test of the CCC.

Results: 2D and M-mode demonstrated excellent and similar intra-observer repeatability, with the best concordance in ES septal thickness (M-mode CCC 0.956 vs 2D-mode CCC 0.914). Interobserver reproducibility demonstrated also a high concordance, optimal in ES left ventricular free wall (M-mode 0.925 vs $2 \mathrm{D}$ 0.855). Comparison of both techniques demonstrated a high concordance in all measurements, except for ED septal thickness with better reproducibility using M-mode (CCC 0.954 vs $0.847, p=0.017)$.
\end{abstract}

Conclusions: 2D and M-mode can be used in a reproducible manner for measuring fetal myocardial thickness, with a slightly better performance of M-mode for assessing ED septal wall thickness. 


\section{INTRODUCTION}

Fetal echocardiography has evolved remarkably during last decades. Initially, its use was limited to assess cardiac structural integrity. ${ }^{1-3}$ However, advances in ultrasound and incorporation of new myocardial imaging techniques permitted a more accurate evaluation of fetal cardiac structure and function. Recently, different patterns of cardiac dysfunction and remodeling have been described in fetal diseases such as twin-to-twin transfusion syndrome, ${ }^{4,5}$ intrauterine growth restriction, ${ }^{6-10}$ congenital heart disease, ${ }^{11,12}$ maternal diabetes, ${ }^{13-16}$ the use of anti-retroviral drugs ${ }^{17}$ or assisted reproductive technologies. ${ }^{18,19}$ An accurate and reproducible measurement of atrial and ventricular structures is essential for an adequate evaluation of adaptive changes in fetal cardiac shape and structure, also known as fetal cardiac remodeling.

Initial studies standardizing fetal cardiac morphometry were based in M-mode ${ }^{1,20}$ and allowed the description of ventricular hypertrophy (i.e. septal hypertrophy in fetuses of diabetic mothers ${ }^{13-16}$ ) or dilatation (i.e. antenatal dilated cardiomyopathy ${ }^{21,22}$ ). Later, improvement of ultrasound resolution and its widespread use in obstetrics have led to the incorporation of 2 dimensional (2D) mode as a fundamental part of fetal echocardiography including its use for cardiac morphometry. ${ }^{23,24}$ However, the technique proposed for the measurement of myocardial thickness differs across and within studies, ${ }^{23-25}$ with no international consensus so far. Additionally, no previous studies have directly compared $2 \mathrm{D}$ and $\mathrm{M}$-mode for measuring of fetal ventricular wall thickness.

The objective of the present study was to evaluate and compare the reproducibility of $2 \mathrm{D}$ versus Mmode for the measurement of fetal myocardial thicknesses. For this, a reproducibility study was conducted in a low-risk population of fetuses at 18-41 weeks of pregnancy. 


\section{METHODS}

Study population

A prospective study including uncomplicated singleton pregnancies between $18+0$ and $41+0$ weeks recruited from low-risk pregnancies attended at BCNatal in Barcelona between October 2015 and October 2016. Exclusion criteria included cases with pre-gestational diabetes mellitus, maternal hypertrophic or dilated cardiomyopathy, preeclampsia, maternal HIV, stillbirth, intrauterine growth restriction, preterm delivery, major congenital birth defect, aneuploidies or genetic syndromes. Gestational age was calculated according to first trimester's crown-rump length. Birthweight centiles were calculated according to local standards. ${ }^{26}$ The study protocol was approved by hospital's Ethical Committee and written consent form was obtained from all participants.

\section{Study protocol and echocardiography}

Demographic and perinatal data were obtained by parental questionnaire and review of hospital records. All participants underwent a single fetal cardiac ultrasound evaluation using a Siemens $®$ Sonoline Antares machine (Siemens Medical Systems, Malvern, PA, USA), with a $2-6 \mathrm{MHz}$ linear curved-array prove and a $2-10 \mathrm{MHz}$ phased-array cardiology prove. A standard fetal echocardiography was first conducted to rule out the presence of any cardiac structural anomaly. Then, M-mode and 2D were applied for measuring myocardial wall thickness at septal and free walls of left and right ventricles.

For M-mode measurements, a four-chamber lateral view was obtained and M-mode was applied and adjusted to include only the fetal heart. The position of the M-mode line was located perpendicular to the septal wall at the level of the atrioventricular valves excursion (Figure 1). The M-mode image obtained was considered adequate for analysis if at least three consecutive heart cycles without fetal movements were present. End-diastole was considered at the largest ventricular cavity diameter just after the closure of the AV valves and end-systole at the narrowest ventricular cavity just before the opening of the $\mathrm{AV}$ valves. Myocardial thickness was measured positioning the calipers following the edges of endo and epicardium.

For 2D evaluation, a movie clip of a lateral four-chamber view, including at least three complete heart cycles without fetal movements, was stored for off-line analysis. Myocardial wall thickness was measured at the level of the atrioventricular valves excursion (Figure 2). For the left and right ventricular walls, caution was taken not to include the chordae tendinae to avoid a false thicker wall. Heart cycle periods were identified with the use of cine-loop. End-diastole was considered at the largest ventricular distention just after the atrioventricular valves closure and end-systole period at the smallest ventricular cavity just before the atrioventricular valves open. 
All measurements were performed off-line by two observers (L.G-O. and A.S-M.) for the interobserver reproducibility. For the intra-observer repeatability, a second analysis was performed by a single observer, at least one week after the first measurement.

Sample size estimation

To determine the adequate sample size for repeatability and reproducibility with continuous variables, the formula proposed by Bonnet DG et al was used. ${ }^{27}$ For an estimated Concordance Correlation Coefficient (CCC) between 0.8 and 0.85 , a sample size of 42 pairs were needed.

Statistical analysis

STATA® 14.2 (Statacorp, College Station, Texas, USA) was used for the statistical analysis. Pvalues less than 0.05 were considered significant. For continuous variables, a Shapiro-Wilk test was used for determination of the distribution. Data was expressed as mean (standard deviation) for normal distributions or median (interquartile range) for non-normal distributions. Categorical variables were expressed as absolute numbers and percentages.

Inter and intra-observer reproducibility was assessed using the Concordance Correlation Coefficient (CCC) ${ }^{28}$ Limits of agreement and standard error were calculated for all comparisons and BlandAltman plots were obtained. ${ }^{29}$ For $2 \mathrm{D}$ vs. M-mode comparison, CCC coefficients were compared with a t-test analysis. To evaluate if the difference between pairs of measurements varies with gestational age, a linear regression analysis was performed. 


\section{RESULTS}

Study population

Image dataset of 45 patients were used. Maternal and perinatal characteristics of patients are described in Table 1. Mean maternal age and birthweight were 32.5 years and $3485 \mathrm{~g}$, respectively. Median gestational age at ultrasound was 29 weeks with the following patient's distribution: 18 to 25 weeks $(n=15), 26$ to 33 weeks $(n=15)$ and 34 to 41 weeks $(n=15)$.

M-mode and $2 D$ reproducibility

Mean values of the fetal ventricular walls were similar between M-mode and 2D-mode (Table 2). Intra-observer analysis demonstrated good repeatability of both septal and left ventricular walls, irrespective of the technique used, with the best concordance in end-systolic septal thickness (Mmode CCC 0.956 and 2D CCC 0.914) and worse performance at the end-diastolic right free ventricular wall (M-mode CCC 0.691 and 2D CCC 0.783) (Table 3). Both techniques showed a similar inter-observer reproducibility (Table 4, Figure 3). Comparison of M-mode vs 2D reproducibility showed a similar performance of both techniques, except for a significantly better intra-observer reproducibility in end-diastolic septal wall thickness by M-mode as compared to 2Dmode (Table 5, Figure 4). Differences between pairs were similar across gestational age ( $\beta$ coefficient $\approx 0 ; p>0.1$ ). 


\section{Discussion}

This study shows that M-mode and 2D are both highly reproducible methods for assessing fetal myocardial wall thicknesses, with slightly better performance of M-mode for end-diastolic septal wall thickness.

Regarding M-mode, our data is in agreement with previous studies reporting a good reproducibility for measuring ventricular wall thickness at end-diastole. ${ }^{20,30-32}$ For instance, Veille et al described a low intra and inter-observer variability of left and right free-walls with M-mode, with values of $\pm 5.1 \%$ and $\pm 4.5 \%$, respectively. ${ }^{20}$ In addition, St. John Sutton et al reported a high inter-observer correlation of 0.94 for left and right ventricular walls. ${ }^{30}$ Regarding septal wall, Macklon et al performed an intra-class correlation analysis in gestational diabetes, with values of 0.71 for both intra and inter-observer reproducibility in fetal septal wall thickness. ${ }^{31}$ The results from the present study also demonstrate a very good reproducibility by M-mode for both free and septal myocardial walls at end-diastole. We further demonstrate that intra and inter-observer reproducibility is also satisfactory when assessed at end-systole, which might be potentially useful for assessing myocardial thickening. In addition, both $2 \mathrm{D}$ and M-mode showed a better repeatability at septal and left ventricular free walls as compared to right free wall -potentially more challenging to assess due to its trabeculation or complex shape-. Interestingly, a recent study of Tedesco et al assessed the inter and intra-observer reproducibility of ventricular walls thickness based on STIC-M mode analysis. This study reported CCC for septal thickness of 0.85 (95\% LoA $-0.056-0.044)$ and 0.86 (95\% LoA $-0.02-0.03)$ for intra and inter-observer reproducibility, similar to the performance achieved by conventional M-mode. ${ }^{32}$

Although most studies report good reproducibility, the methodology reported to measure myocardial thickness with M-mode is variable. While many studies used only a lateral four-chamber view approach, ${ }^{33,34}$ others also use short axis view, in cases of suboptimal visualization of fetal heart. ${ }^{1}$ Even more, despite that M-line is commonly placed using the open AV-valves plane, ${ }^{1,20,33,34}$ some groups have described the use of midventricular level at end-diastole. ${ }^{32}$ In our study, the method and reference landmarks for M-mode were rigorously defined based on latest fetal echocardiographic studies ${ }^{33,34}$ and also on adult's cardiology Guidelines. ${ }^{35}$ Briefly, we recommend the use of a lateral four-chamber view, with M-mode applied at the level of AV-valves excursion, and measurements of ventricular walls performed when the widest and narrowest ventricular cavity was observed, following the edges of endo and epicardium. In addition, we additionally used 2D to corroborate the correct recognition of ventricular structures when using M-mode. Overall, we believe that the use of strict methodological criteria has enabled to report a very high reproducibility for fetal myocardial walls both at end-diastole and end-systole.

Regarding 2D, we also confirm previous data reporting good inter and intra-observer reproducibility for ventricular walls with intra and inter-observer variabilities of $<5-7 \%$ for both ventricles ${ }^{25}$ Again, 
the methodology used for measuring fetal myocardial walls thickness -mainly in septum- by 2D varies among studies. ${ }^{15,23,25,36}$ While, some studies reported measurements in an apical fourchamber view at end-diastole below coapted of atrioventricular leaflets, ${ }^{23}$ others used a lateral four chamber view. ${ }^{15}$ In addition, the proposed place to measure the septum also varies from using atrioventricular leaflets as a reference landmark ${ }^{25}$ to a mid-septum approach. ${ }^{36}$ In the present study, we propose a lateral four-chamber view to achieve a better identification of the chordae tendinae. Furthermore, all measurements were performed at the level of the atrioventricular valves excursion to avoid the inclusion of the valve in the wall thickness. The use of the atrioventricular valves excursion should allow us a better inter and intra-observer reproducibility, because of a well-defined reference landmark. Furthermore, based on our experience, we consider that the exclusion of chordae tendinae is very important to avoid a false overestimation of lateral walls thickness, which are difficult to identify during end-diastole, and commonly measured as part of the lateral wall. To avoid this, the use of cine-loop is useful to track the wall displacement. Another important aspect to consider is that ultrasound is characterized by a better axial than lateral resolution, which allows a good definition of close structures. ${ }^{24}$

Despite many studies having previously evaluated the performance of M-mode and 2D, to our knowledge, this is the first study focused in a direct comparison of both techniques. Our study demonstrates a similar reproducibility for $\mathrm{M}$-mode and $2 \mathrm{D}$ when assessing ventricular wall thicknesses. Interestingly, M-mode appears to have a slightly better repeatibility for septal wall thickness at end-diastole. This finding could be related to a better identification of the limits of ventricular structures across the cardiac cycle when M-mode is applied.

From a clinical perspective, the assessment of ventricular walls thickness in fetal life has gained relevance since the description of antenatal cardiac remodeling as an adaptive mechanism to compensate a noxa. ${ }^{37}$ Several antenatal insults, such as hypoxia, lack of nutrients, infection or inflammation could affect the developing heart that adapts by changing its shape and function. ${ }^{17,38}$ For example, maternal diabetes mellitus has been widely described to associate fetal myocardial hypertrophy (particularly thickenning of the basal septal wall). ${ }^{31}$ More recently, fetal cardiac hypertrophy has also been related to the use of the maternal anti-retroviral treatment with zidovudine in HIV infected pregnancies. ${ }^{17}$ Intrauterine growth restriction has also been associated with changes in fetal cardiac shape -more spherical ventricles- together myocardial hypertrophy in the more severe cases. ${ }^{6,10}$

Current guidelines for echocardiography in adult's population proposed the use of septal enddiastolic thickness and the estimated ventricular mass (either with M-mode or 2D-mode) for assessing and quantifying the presence of cardiac hypertrophy. ${ }^{35}$ ISUOG Guidelines for fetal heart assessment mention the use of M-mode as a good method for ventricular walls thickness, but the 
optimal methodology is not detailed. ${ }^{39}$ Based on our results, we propose a well-defined technique for measuring fetal myocardial wall thickness in order to achieve an optimal performance for assessing fetal myocardial thickness. In summary, a lateral four-chamber view should be obtained for both $2 \mathrm{D}$ and $\mathrm{M}$ mode, with clear visualization of the excursion of both $\mathrm{AV}$ valves. For $2 \mathrm{D}$ approach, walls thickness should be measured at the level of the atrioventricular valves excursion, with the largest ventricular distension for end-diastole just after the atrioventricular valves closure, and the smaller volume for end-systole, just before the atrioventricular valves opening. (Figure 1). For M-mode, the reference M-line should be applied perpendicular to the septum, also at the level of the atrioventricular valves excursion, and measurements considering the widest and narrowest ventricular diameter (Figure 2). In addition, we believe that both, 2D or M-mode could be used depending of the operator's experience or preferences, although a slightly better performance could be achieved by M-mode.

The present study has several strengths and limitations that merit comment. Firstly, we used a rigorous and well-defined technique for both $\mathrm{M}$ and $2 \mathrm{D}$ mode measurements. The definition of a well-defined method, using clear anatomic references in both M-mode and 2D-mode might be optimal for developing reference ranges, and for clinical application in conditions leading to fetal cardiac remodeling. Secondly, to our knowledge, this is the first study focused in comparing the use of $2 \mathrm{D}$ and $\mathrm{M}$-mode in the same population, both at end-diastole and end-systole, and demonstrating for the first time a slightly better performance of M-mode as compared to $2 \mathrm{D}$ for septal wall thickness. However, this study has limitations. We acknowledge that the use of normal healthy pregnancies could affect the external validation in fetal conditions related to cardiac affection. Future studies are warranted to confirm and validate our results in fetal or maternal pathologies. In conclusion, our study demonstrated that both M-mode and 2D-mode are good and reproducible techniques for the assessment of fetal ventricular walls thickness, with a slightly better performance of M-mode for septal wall thickness. The choice could be based on the operator's experience and the fetal or maternal condition. 


\begin{abstract}
Aknowledgment
This project has been co-funded with support of the Erasmus Mundus Joint Doctorate in Fetal and Perinatal Medicine Programme of the European Union (Framework Agreement number: 20130040). This publication reflects the views only of the author, and the Commission cannot be held responsible for any use which may be made of the information contained therein. Additionally, the research leading to these results has received funding from "la Caixa" Foundation; Cerebra Foundation for the Brain Injured Child (Carmarthen, Wales, UK); the Instituto de Salud Carlos III (PI14/00226, PI15/00263, PI15/00130 and INT16/00168) as part of the Plan Nacional de I+D+I and co-funded by ISCIII-Subdirección General de Evaluación y el Fondo Europeo de Desarrollo Regional (FEDER) "Otra manera de hacer Europa" (Spain) and the support of the Secretaria d'Universitat i Recerca del Departament d'Economia i Coneixement de la Generalitat de Catalunya (Grant SGR n928 and 2016FI_B01184). Iris Soveral was supported by a grant (Ajut Josep Font) from Hospital Clinic Barcelona.
\end{abstract}




\section{References}

1. Allan LD, Joseph MC, Boyd EGCA, Campbell S, Tynan M. M-mode echocardiography in the developing human fetus. Br Hear J. 1982;47:573-583.

2. Sharland GK, Allan LD. Normal fetal cardiac measurements derived by cross-sectional echocardiography. Ultrasound Obstet Gynecol. 1992;2:175-181.

3. Shapiro I, Degani S, Leibovitz Z, et al. Fetal cardiac measurements derived by transvaginal and transabdominal cross-sectional echocardiography from 14 weeks of gestation to term. Ultrasound Obstet Gynecol. 1998;12:404-418.

4. Votava-Smith JK, Habli M, Cnota JF, Divanovic A, et al. Diastolic dysfunction and cerebrovascular redistribution precede overt recipient twin cardiomyopathy in early-stage twin-twin transfusion syndrome. J Am Soc Echocardiogr. 2015;28(5):533-540.

5. Wohlmuth C, Boudreaux D, Moise KJJ, et al. Cardiac pathophysiology in twin-twin transfusion syndrome: New insights into its evolution. Ultrasound Obstet Gynecol. March 2017.

6. Rodriguez-Lopez M, Cruz-Lemini M, Valenzuela-Alcaraz B, et al. Descriptive analysis of the different phenotypes of cardiac remodeling in fetal growth restriction. Ultrasound Obstet Gynecol. November 2016:[Epub ahead of print].

7. Crispi F, Bijnens B, Sepulveda-Swatson E, et al. Post-Systolic Shortening by Myocardial Deformation Imaging as a Sign of Cardiac Adaptation to Pressure Overload in Fetal Growth Restriction. Circ Cardiovasc Imaging. 2014;7(5):781-787.

8. Cruz-Lemini M, Crispi F, Valenzuela-Alcaraz B, et al. Value of annular M-mode displacement vs tissue Doppler velocities to assess cardiac function in intrauterine growth restriction. Ultrasound Obstet Gynecol. 2013;42(2):175-181.

9. Comas M, Crispi F, Cruz-Martinez R, et al. Usefulness of myocardial tissue Doppler vs conventional echocardiography in the evaluation of cardiac dysfunction in early-onset intrauterine growth restriction. Am J Obstet Gynecol. 2010;203(1):45.e1-45.e7.

10. Cruz-Lemini M, Crispi F, Valenzuela-Alcaraz B, et al. A fetal cardiovascular score to predict infant hypertension and arterial remodeling in intrauterine growth restriction. Am J Obstet Gynecol. 2014;210(6):552.e1-552.e22.

11. Miranda JO, Hunter L, Tibby S, et al. Myocardial deformation in fetuses with coarctation of the aorta: a case-control study. Ultrasound Obstet Gynecol. 2017;49(5):623-629.

12. Guirado L, Crispi F, Masoller N, et al. Biventricular impact of mild to moderate pulmonary 
valve stenosis in fetal life. Ultrasound Obstet Gynecol. March 2017.

13. Balli S, Pac FA, Oflaz MB, et al. Assessment of Cardiac Functions in Fetuses of Gestational Diabetic Mothers. Pediatr Cardiol. 2014;35:30-37.

14. Wang $\mathrm{H}, \mathrm{Xu} \mathrm{Y,} \mathrm{Fu} \mathrm{J,} \mathrm{et} \mathrm{al.} \mathrm{Evaluation} \mathrm{of} \mathrm{the} \mathrm{regional} \mathrm{ventricular} \mathrm{systolic} \mathrm{function} \mathrm{by} \mathrm{two-}$ dimensional strain echocardiography in gestational diabetes mellitus ( GDM ) fetuses with good glycemic control Evaluation of the regional ventricular systolic function by twodimensional str. J Matern Fetal Neonatal Med. 2015;28(18):2150-2154.

15. Ren $Y$, Zhou Q, Yan $Y$, et al. Characterization of fetal cardiac structure and function detected by echocardiography in women with normal pregnancy and gestational diabetes mellitus †. Prenat Diagn. 2011;31:459-465.

16. Veille JC, Sivakoff M, Hanson R, Fanaroff AA. Interventricular septal thickness in fetuses of diabetic mothers. Obstet Gynecol. 1992;79(1):51-54.

17. García-Otero L, López M, Gómez O, et al. Zidovudine treatment in HIV-infected pregnant women is associated with fetal cardiac remodelling. AIDS. 2016;30(February):1393-1401.

18. Valenzuela-Alcaraz B, Crispi F, Bijnens B, et al. Assisted Reproductive Technologies Are Associated With Cardiovascular Remodeling In Utero That Persists Postnatally. Circulation. 2013;128(13):1442-1450.

19. Valenzuela-Alcaraz B, Cruz-Lemini M, Rodriguez-Lopez M, et al. Fetal cardiac remodeling in twin pregnancies conceived by assisted reproductive technologies. Ultrasound Obstet Gynecol. May 2017:[Epub ahead of print].

20. Veille JC, Sivakoff M, Nemeth M. Evaluation of the human fetal cardiac size and function. Am J Perinatol. 1990;7(1):54-59.

21. Mongiovi M, Fesslova V, Fazio G, et al. Diagnosis and prognosis of fetal cardiomyopathies: a review. Curr Pharm Des. 2010;16(26):2929-2934.

22. Sivasankaran S, Sharland GK, Simpson JM. Dilated cardiomyopathy presenting during fetal life. Cardiol Young. 2005;15(4):409-416.

23. Firpo C, Silverman NH. Evaluation of Fetal Heart Dimensions from 12 Weeks to Term. Am J Cardiol. 2001;87:594-600.

24. Tan J, Silverman NH, Hoffman Jl, et al. Cardiac dimensions determined by cross-sectional echocardiography in the normal human fetus from 18 weeks to term. Am J Cardiol. 1992;70(18):1459-1467.

25. Oberhoffer R, Högel J, Lang D. Normal characteristics of cardiac dimensions and function in 
the fetus. Eur J Ultrasound. 1995;2(2):93-106.

26. Figueras F, Meler E, Iraola A, et al. Customized birthweight standards for a Spanish population. Eur J Obstet Gynecol Reprod Biol. 2008;136(1):20-24.

27. Watson PF, Petrie A. Method agreement analysis : A review of correct methodology. Theriogenology. 2010;73(9):1167-1179.

28. Lin LI. A concordance correlation coefficient to evaluate reproducibility. Biometrics. 1989;45(1):255-268.

29. Bland JM, Altman DG. Applying the right statistics : analyses of measurement studies. Ultrasound Obstet Gynecol. 2003;22:85-93.

30. St John Sutton MG, Gewitz MH, Shah B, et al. Quantitative assessment of growth and function of the cardiac chambers in the normal human fetus: a prospective longitudinal echocardiographic study. Circulation. 1984;69(4):645-654.

31. Macklon NS, Hop WC, Wladimiroff JW. Fetal cardiac function and septal thickness in diabetic pregnancy: a controlled observational and reproducibility study. Br J Obstet Gynaecol. 1998;105(6):661-666.

32. Tedesco GD, de Souza Bezerra M, Barros FS, et al. Reference Ranges of Fetal Cardiac Biometric Parameters Using Three-Dimensional Ultrasound with Spatiotemporal Image Correlation M Mode and Their Applicability in Congenital Heart Diseases. Pediatr Cardiol. 2017;38:271-279.

33. Luewan S, Yanase $\mathrm{Y}$, Tongprasert F, et al. Fetal cardiac dimensions at 14-40 weeks ' gestation obtained using cardio-STIC-M. Ultrasound Obstet Gynecol. 2011;37:416-422.

34. Devore GR. Assessing fetal cardiac ventricular function. Semin Fetal Neonatal Med. 2005;10:515-541.

35. Lang RM, Badano LP, Mor-Avi V, et al. Recommendations for Cardiac Chamber Quantification by Echocardiography in Adults: An Update from the American Society of Echocardiography and the European Association of Cardiovascular Imaging. J Am Soc Echocardiogr. 2015;28(1):1-39.e14.

36. Garcia-flores J, Jañez M, Cruz Gonzalez M, et al. Fetal myocardial morphological and functional changes associated with well-controlled gestational diabetes. Eur J Obstet Gynecol Reprod Biol. 2011;154:24-26.

37. Crispi F, Bijnens B, Figueras F, et al. Fetal Growth Restriction Results in Remodeled and Less Efficient Hearts in Children. Circulation. 2010;121(22):2427-2436. 
38. Cruz-Lemini M, Crispi F, Valenzuela-Alcaraz B, et al. Fetal cardiovascular remodeling persists at 6 months in infants with intrauterine growth restriction. Ultrasound Obstet Gynecol. 2016;48(3):349-356.

39. Carvalho J, Allan L, Chaoui R, et al. isuog Practice Guidelines (updated): sonographic screening examination of the fetal heart. Ultrasound Obstet Gynecol. 2013;41:348-359.

http://mc.manuscriptcentral.com/pd 


\section{Figure legends.}

Figure 1. Description of landmarks for ventricular walls assessment by 2D-mode.

a) Four-chamber lateral view at end-diastole. Left image: 2D-ultrasound; right image: descriptive scheme; b) Four-chamber lateral view at end-systole. Left image: 2D-ultrasound; right image: descriptive scheme of $2 \mathrm{D}$ measurement.

$\mathrm{RV}=$ right ventricle; $\mathrm{S}=$ septal wall; $\mathrm{LV}=$ left ventricle; $\mathrm{CT}=$ chordae tendinae.

Figure 2. Description of landmarks for ventricular walls assessment by M-mode.

Upper-left image: 2D-ultrasound of a lateral four-chamber view. White thick line corresponds to reference M-line. Lower-left image: M-mode representation of the reference M-line. Lower-right image: descriptive scheme of M-mode measurement.

$\mathrm{RV}=$ right ventricle; $\mathrm{S}=$ septal wall; $\mathrm{LV}=$ left ventricle; $\mathrm{CT}=$ chordae tendinae; $1=\mathrm{Left}$ ventricular wall thickness at end-systole; $2=$ Septal wall thickness at end-systole; $3=$ Right ventricular wall thickness at end-systole; $4=$ Left ventricular wall thickness at end-diastole; $5=$ Septal wall thickness at enddiastole; $6=$ Right ventricular wall thickness at end-diastole

Figure 3. Bland-Altman plots for intra - observer reproducibility of left, right and septal walls thickness with M-mode versus 2D-mode.

a) Intra - observer reproducibility of septal thickness at end-diastole; b) Intra - observer reproducibility of left ventricular wall thickness at end-diastole; c) Intra - observer reproducibility of right ventricular wall thickness at end-diastole.

Figure 4. Bland-Altman plots for inter - observer reproducibility of left, right and septal walls thickness with M-mode versus 2D-mode.

a) Inter - observer reproducibility of septal thickness at end-diastole; b) Inter - observer reproducibility of left ventricular wall thickness at end-diastole; c) Inter - observer reproducibility of right ventricular wall thickness at end-diastole. 
Table 1. Maternal and perinatal characteristics of the study population.

\begin{tabular}{lc}
\hline Variable & Value \\
\hline Maternal characteristics & $32.5 \pm 4.63$ \\
Age, years & $22.7 \pm 2.86$ \\
Body mass index, $\mathrm{kg} / \mathrm{m}^{2}$ & 0 \\
Chronic diseases & $4(8.9)$ \\
Smoking habit & $24(53.3)$ \\
Nulliparous & $1(2.2)$ \\
\hline Perinatal characteristics & $40.3(39.5-41.0)$ \\
Cesarean section & $3485(3230-3841)$ \\
Gestational age at birth, weeks & $70.5(22.5-86.0)$ \\
Birthweight, grams & 0 \\
Birthweight percentile & \\
5-minutes APGAR $<7$ & \\
\hline Data expressed as mean \pm standard deviation, median (interquartile range) or $\mathrm{n}(\%)$.
\end{tabular}

http://mc.manuscriptcentral.com/pd 
Table 2. Mean values for fetal myocardial wall thicknesses using $2 \mathrm{D}$ and $\mathrm{M}$-mode.

\begin{tabular}{lccc}
\hline Variable & M-MODE & 2D & p-value \\
\hline Septum (d) & $3.08 \pm 0.96$ & $2.87 \pm 0.87$ & 0.27 \\
Septum (s) & $4.06 \pm 1.23$ & $3.63 \pm 1.03$ & 0.07 \\
LV free wall (d) & $2.77 \pm 0.84$ & $2.55 \pm 0.80$ & 0.21 \\
LV free wall (s) & $3.38 \pm 0.94$ & $3.08 \pm 0.91$ & 0.13 \\
RV free wall (d) & $2.61 \pm 0.79$ & $2.66 \pm 0.85$ & 0.79 \\
RV free wall (s) & $3.21 \pm 0.98$ & $3.13 \pm 0.90$ & 0.70 \\
\hline
\end{tabular}

All the measurements in $\mathrm{mm}$ and expressed as mean \pm SD.

$\mathrm{P}$-value calculated by t-test comparison of mean values by $2 \mathrm{D}$ vs $\mathrm{M}$-mode.

$\mathrm{LV}=$ left ventricular, $\mathrm{RV}=$ right ventricular, $(\mathrm{d})=$ end-diastolic, $(\mathrm{s})$ end-systolic. 
Table 3. Intra-observer reproducibility for fetal myocardial wall thickness measurements by M-mode and $2 \mathrm{D}$.

\begin{tabular}{lcccc}
\hline \multicolumn{1}{c}{ Variable } & CCC & Standard error & $\begin{array}{c}95 \% \text { confidence } \\
\text { interval }\end{array}$ & $\begin{array}{c}\text { 95\% limits of } \\
\text { agreement }\end{array}$ \\
\hline Septum (d) & 0.954 & 0.014 & $0.928-0.981$ & $-0.551-0.595$ \\
Septum (s) & 0.956 & 0.013 & $0.930-0.982$ & $-0.655-0.757$ \\
LV free wall (d) & 0.859 & 0.038 & $0.784-0.934$ & $-0.542-0.987$ \\
LV free wall (s) & 0.935 & 0.019 & $0.898-0.972$ & $-0.476-0.738$ \\
RV free wall (d) & 0.691 & 0.067 & $0.559-0.823$ & $-0.532-1.309$ \\
RV free wall (s) & 0.834 & 0.043 & $0.751-0.917$ & $-0.747-1.151$ \\
& & $2 \mathrm{D}-\mathrm{MODE}$ & & \\
Septum (d) & 0.847 & 0.042 & $0.765-0.930$ & $-1.028-0.940$ \\
Septum (s) & 0.914 & 0.025 & $0.866-0.963$ & $-0.841-0.890$ \\
LV free wall (d) & 0.877 & 0.035 & $0.808-0.946$ & $-0.826-0.760$ \\
LV free wall (s) & 0.905 & 0.027 & $0.851-0.959$ & $-0.774-0.774$ \\
RV free wall (d) & 0.783 & 0.059 & $0.668-0.898$ & $-1.010-1.121$ \\
RV free wall (s) & 0.868 & 0.037 & $0.795-0.941$ & $-0.996-0.832$
\end{tabular}

$\mathrm{CCC}=$ concordance correlation coefficient; $\mathrm{LV}=$ left ventricular; $\mathrm{RV}=$ right ventricular; $(\mathrm{d})=$ enddiastole; (s)= end-systole. 
Table 4. Inter-observer reproducibility for fetal myocardial wall thickness measurements by M-mode versus $2 \mathrm{D}$.

\begin{tabular}{lcccc}
\hline Variable & CCC & Standard error & $\begin{array}{c}95 \% \text { confidence } \\
\text { interval }\end{array}$ & $\begin{array}{c}\text { 95\% limits of } \\
\text { agreement }\end{array}$ \\
\hline \multicolumn{5}{c}{ M-MODE } \\
Septum (d) & 0.827 & 0.048 & $0.733-0.920$ & $-0.920-1.258$ \\
Septum (s) & 0.745 & 0.058 & $0.631-0.859$ & $-0.644-1.920$ \\
LV free wall (d) & 0.890 & 0.031 & $0.830-0.950$ & $-0.876-0.752$ \\
LV free wall (s) & 0.925 & 0.022 & $0.883-0.967$ & $-0.707-0.769$ \\
RV free wall (d) & 0.830 & 0.047 & $0.737-0.923$ & $-0.931-0.900$ \\
RV free wall (s) & 0.871 & 0.037 & $0.799-0.942$ & $-0.920-0.978$ \\
& & $2 D-M O D E$ & \\
Septum (d) & 0.852 & 0.042 & $0.770-0.933$ & $-0.947-0.956$ \\
Septum (s) & 0.791 & 0.053 & $0.688-0.895$ & $-0.716-1.494$ \\
LV free wall (d) & 0.823 & 0.049 & $0.726-0.919$ & $-0.940-0.940$ \\
LV free wall (s) & 0.855 & 0.040 & $0.776-0.934$ & $-0.796-1.089$ \\
RV free wall (d) & 0.727 & 0.071 & $0.587-0.867$ & $-1.129-1.315$ \\
RV free wall (s) & 0.805 & 0.052 & $0.703-0.908$ & $-0.916-1.285$
\end{tabular}

$\overline{\mathrm{CCC}}=$ concordance correlation coefficient; $\mathrm{LV}=$ left ventricular; $\mathrm{RV}=$ right ventricular; $(\mathrm{d})=$ enddiastole; (s)= end-systole. 
Table 5. Comparison of M-mode and 2D-mode intra- and inter-observer reproducibility for fetal myocardial wall thicknesses.

\begin{tabular}{lccccc}
\hline \multicolumn{1}{c}{ Variable } & CCC & $\begin{array}{c}\text { Standard } \\
\text { error }\end{array}$ & $\begin{array}{c}95 \% \text { Confidence } \\
\text { interval }\end{array}$ & $\begin{array}{c}95 \% \text { limit of } \\
\text { agreement }\end{array}$ & p-value* \\
\hline \multicolumn{1}{c}{ Inter-observer } & & & & & \\
Septum (d) & 0.809 & 0.036 & $0.738-0.879$ & $-0.986-1.235$ & 0.70 \\
Septum (s) & 0.763 & 0.041 & $0.682-0.845$ & $-1.131-1.758$ & 0.56 \\
LV free wall (d) & 0.780 & 0.040 & $0.702-0.857$ & $-0.763-1.261$ & 0.25 \\
LV free wall (s) & 0.793 & 0.037 & $0.721-0.855$ & $-0.666-1.377$ & 0.13 \\
RV free wall (d) & 0.757 & 0.045 & $0.668-0.846$ & $-1.109-1.138$ & 0.23 \\
RV free wall (s) & 0.804 & 0.037 & $0.732-0.877$ & $-0.949-1.286$ & 0.30 \\
\hline$\quad$ Intra-observer & & & & & \\
Septum (d) & 0.842 & 0.043 & $0.758-0.925$ & $-0.720-1.146$ & 0.017 \\
Septum (s) & 0.810 & 0.052 & $0.708-0.912$ & $-0.991-1.284$ & 0.14 \\
LV free wall (d) & 0.784 & 0.057 & $0.673-0.895$ & $-0.773-1.209$ & 0.7 \\
LV free wall (s) & 0.839 & 0.042 & $0.756-0.922$ & $-0.579-1.175$ & 0.37 \\
RV free wall (d) & 0.769 & 0.062 & $0.648-0.891$ & $-1.134-1.040$ & 0.3 \\
RV free wall (s) & 0.821 & 0.049 & $0.725-0.917$ & $-1.017-1.173$ & 0.55 \\
\hline
\end{tabular}

$\mathrm{CCC}=$ concordance correlation coefficient between M-mode and 2D; $(\mathrm{d})=$ end-diastole; $(\mathrm{s})=$ endsystole; $L V=$ left ventricular; $R V=$ right ventricular.

${ }^{*} \mathrm{P}$-value calculated by t-test comparing CCC coefficients of M-mode and 2D. 


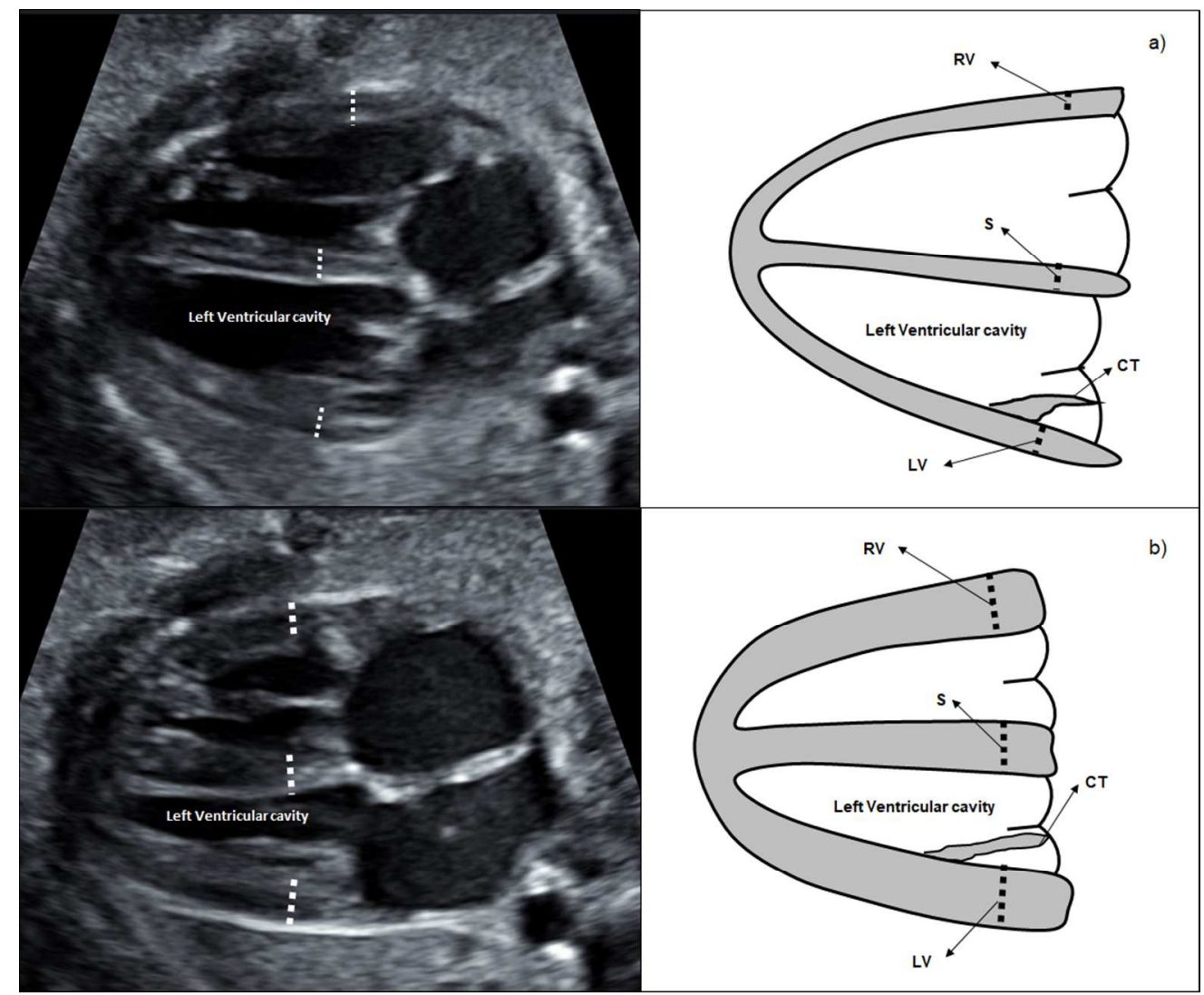

Figure 1. Description of landmarks for ventricular walls assessment by 2D-mode.

a) Four-chamber lateral view at end-diastole. Left image: 2D-ultrasound; right image: descriptive scheme; b) Four-chamber lateral view at end-systole. Left image: 2D-ultrasound; right image: descriptive scheme of 2D measurement.

$\mathrm{RV}=$ right ventricle; $\mathrm{S}=$ septal wall; $\mathrm{LV}=$ left ventricle; $\mathrm{CT}=$ chordae tendinae. 


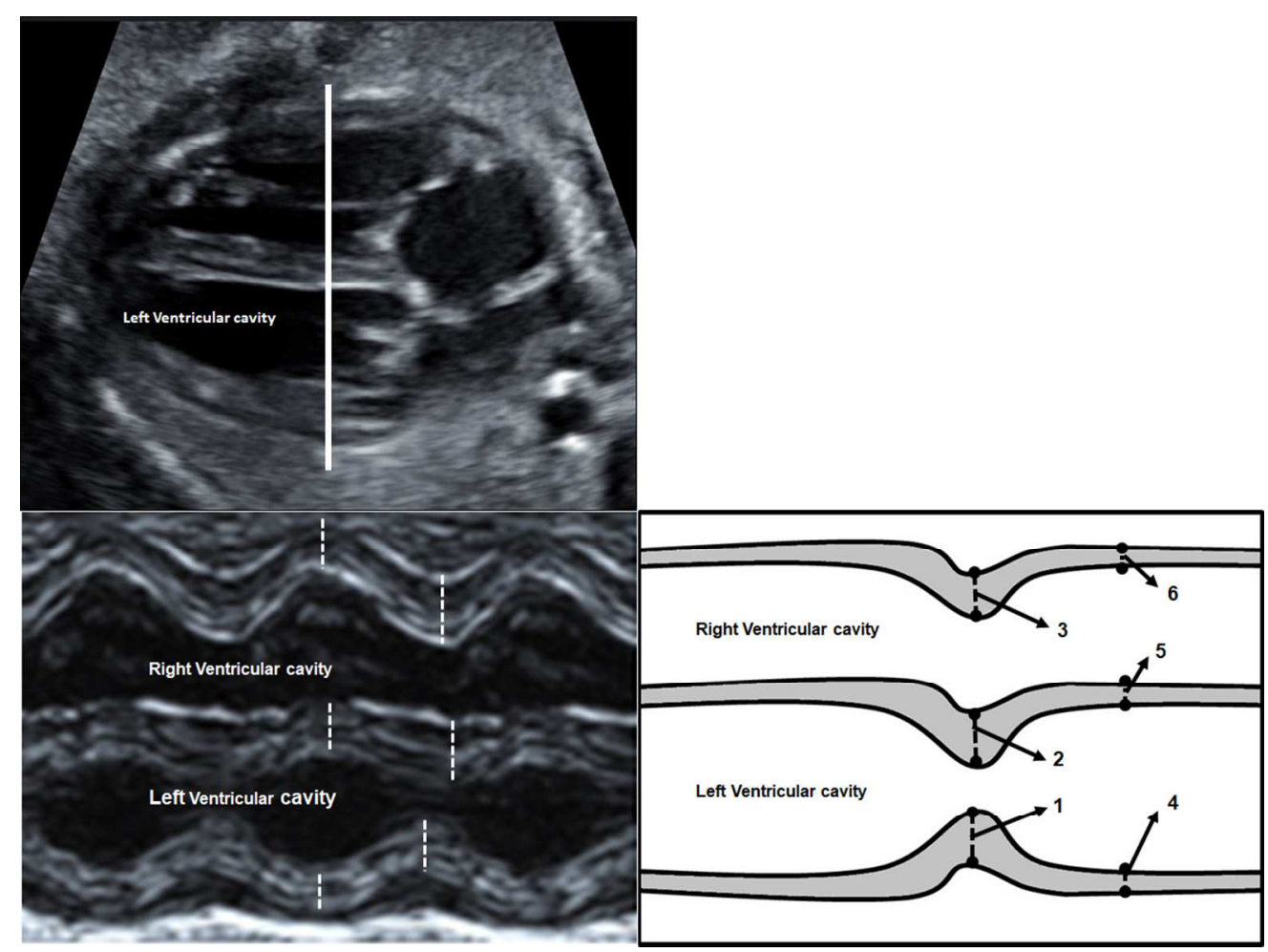

Figure 2. Description of landmarks for ventricular walls assessment by M-mode.

Upper-left image: 2D-ultrasound of a lateral four-chamber view. White thick line corresponds to reference M-line. Lower-left image: M-mode representation of the reference M-line. Lower-right image: descriptive scheme of M-mode measurement.

$\mathrm{RV}=$ right ventricle; $\mathrm{S}=$ septal wall; $\mathrm{LV}=$ left ventricle; $\mathrm{CT}=$ chordae tendinae $1=$ Left ventricular wall thickness at end-systole; 2 =Septal wall thickness at end-systole; $3=$ Right ventricular wall thickness at end-systole; $4=$ Left ventricular wall thickness at end-diastole; $5=$ Septal wall thickness at end-diastole; $6=$ Right ventricular wall thickness at end-diastole 

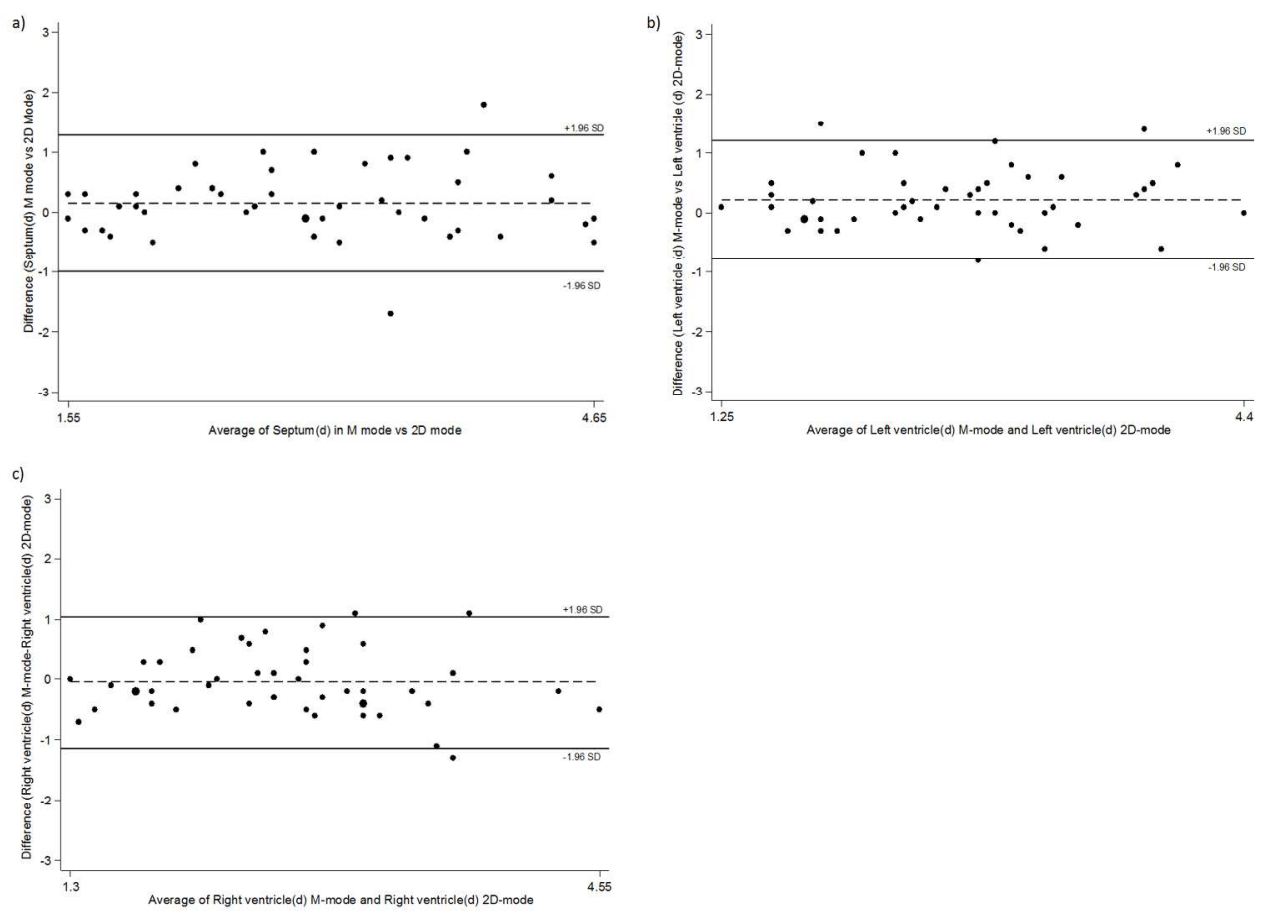

Figure 3. Bland-Altman plots for intra - observer reproducibility of left, right and septal walls thickness with M-mode versus 2D-mode.

a) Intra - observer reproducibility of septal thickness at end-diastole; b) Intra - observer reproducibility of left ventricular wall thickness at end-diastole; c) Intra - observer reproducibility of right ventricular wall thickness at end-diastole. 

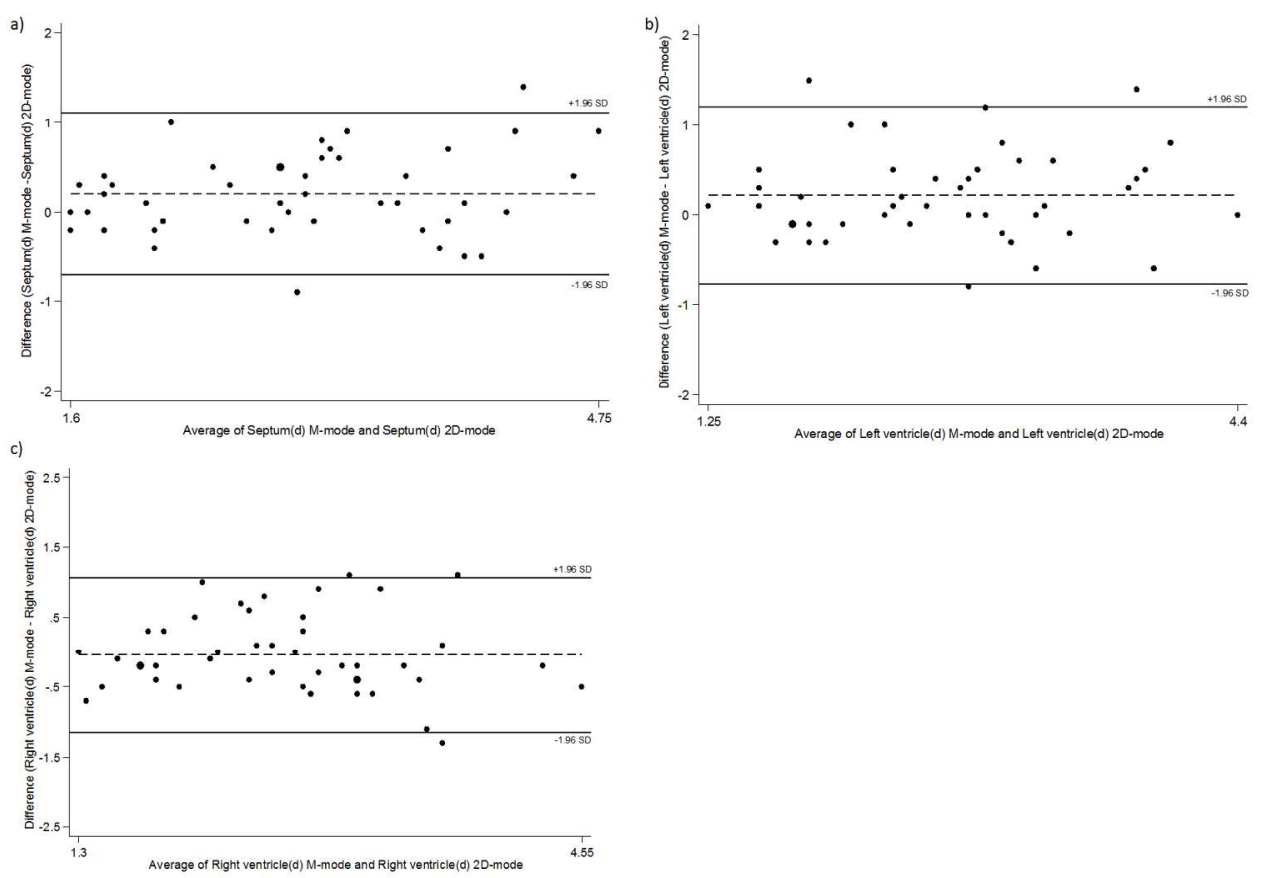

Figure 4. Bland-Altman plots for inter - observer reproducibility of left, right and septal walls thickness with M-mode versus 2D-mode.

a) Inter - observer reproducibility of septal thickness at end-diastole; b) Inter - observer reproducibility of left ventricular wall thickness at end-diastole; c) Inter - observer reproducibility of right ventricular wall thickness at end-diastole. 